J. Korean Math. Soc. 52 (2015), No. 1, pp. 23-41

http://dx.doi.org/10.4134/JKMS.2015.52.1.023

\title{
EXPANDING THE CONVERGENCE DOMAIN FOR CHUN-STANICA-NETA FAMILY OF THIRD ORDER METHODS IN BANACH SPACES
}

\author{
Ioannis Konstantinos Argyros, Santhosh George, \\ And Ángel Alberto Magreñán
}

\begin{abstract}
We present a semilocal convergence analysis of a third order method for approximating a locally unique solution of an equation in a Banach space setting. Recently, this method was studied by Chun, Stanica and Neta. These authors extended earlier results by Kou, Li and others. Our convergence analysis extends the applicability of these methods under less computational cost and weaker convergence criteria. Numerical examples are also presented to show that the earlier results cannot apply to solve these equations.
\end{abstract}

\section{Introduction}

In this study we are concerned with the problem of approximating a locally unique solution $x^{*}$ of the equation

$$
F(x)=0,
$$

where $F$ is a Fréchet-differentiable operator defined on a convex subset $D$ of a Banach space $X$ with values in a Banach space $Y$.

Many problems in computational mathematics and other disciplines can be brought in a form like (1.1) using mathematical modelling $[2,4,12,15,17$, $20,21]$. The solutions of these equations can rarely be found in closed form. That is why most solution methods for these equations are usually iterative. In particular the practice of Numerical Functional Analysis for finding such solutions is essentially connected to Newton-like methods $[1,2,4,11,12,15,16$, $17,19,20,21]$. The study about convergence of iterative procedures is normally centered on two types: semi-local and local convergence analysis. The semilocal convergence matter is, based on the information around an initial point, to give criteria ensuring the convergence of the iterative procedures. While the local analysis is based on the information around a solution, to find estimates

Received January 30, 2014; Revised April 4, 2014.

2010 Mathematics Subject Classification. 65H10,65G99, 65K10, 47H17, 49M15.

Key words and phrases. family of third order method, Newton-like methods, Banach space, semilocal convergence, majorizing sequences, recurrent relations, recurrent functions. 
of the radii of convergence balls. There exist many studies which deal with the local and the semilocal convergence analysis of Newton-like methods such as [1]-[22].

Majorizing sequences in connection to the Kantorovich theorem have been used extensively for studying the convergence of these methods $[2,3,4,7,12$, 17]. Rall [21] suggested a different approach for the convergence of these methods, based on recurrent relations. Candela and Marquina [5, 6], Parida [18], Parida and Gupta [19], Magreñán [15], Ezquerro and Hernández [8], Gutiérrez and Hernández $[9,10]$. Argyros [2, 3, 4] used this idea for several high-order methods. In particular, Kou and Li [13] introduced a third order family of methods for solving equation (1.1), when $X=Y=\mathbb{R}$ defined by

$$
\begin{gathered}
y_{n}=x_{n}-\theta F^{\prime}\left(x_{n}\right)^{-1} F\left(x_{n}\right) \text { for each } n=0,1,2, \ldots \\
x_{n+1}=x_{n}-\frac{\theta^{2}+\theta-1}{\theta^{2}} F^{\prime}\left(x_{n}\right)^{-1} F\left(x_{n}\right)-\frac{1}{\theta^{2}} F^{\prime}\left(x_{n}\right)^{-1} F\left(y_{n}\right),
\end{gathered}
$$

where $x_{0}$ is an initial point and $\theta \in \mathbb{R}-\{0\}$. This family uses two evaluations of $F$ and one evaluation of $F^{\prime}$. Third order methods requiring one evaluation of $F$ and two evaluation of $F^{\prime}$ can be found in [2, 4, 13, 20]. It is well known that the convergence domain of high order methods is in general very small. This fact limits the applicability of these methods. In the present study we are motivated by this fact and recent work by Chun, Stanica and Neta [7] who provided a semilocal convergence analysis of the third order method (1.2) in a Banach space setting. Their semilocal convergence analysis is based on recurrent relations. In Section 2 we show convergence of the third order method (1.2) using more precise recurrent relations under less computational cost and weaker convergence criterion. Moreover, the error estimates on the distances $\left\|x_{n+1}-x_{n}\right\|,\left\|x_{n}-x^{*}\right\|$ are more precise and the information on the location of the solution at least as precise. In Section 3 using our technique of recurrent functions we present a semilocal convergence analysis using majorizing sequence. The convergence criterion can be weaker than the older convergence criteria or the criteria of Section 2. Numerical examples are presented in Section 4 that show the advantages of our work over the older works.

\section{Semilocal convergence I}

Let $U(w, \rho), \bar{U}(w, \rho)$ stand for the open and closed ball, respectively, with center $w \in X$ and of radius $\rho>0$. Let also $L(X, Y)$ denote the space of bounded linear operators from $X$ into $Y$.

The semilocal convergence analysis of third order method (1.2), given by Chun, Stanica and Neta [7] is based on the following conditions. Suppose:

$(\mathcal{C})$ :

(1) There exists $\left\|F^{\prime}(x)-F^{\prime}(y)\right\| \leq K\|x-y\|$ for each $x$ and $y \in D$;

(2) $\left\|F^{\prime \prime}(x)\right\| \leq M$ for each $x \in D$;

(3) $\left\|F^{\prime}\left(x_{0}\right)^{-1}\right\| \leq \beta$; 
(4) $\left\|F^{\prime}\left(x_{0}\right)^{-1} F\left(x_{0}\right)\right\| \leq \eta$.

They defined certain parameters and sequences by

$$
\begin{aligned}
a & =K \beta \eta, \\
\alpha & =\frac{\left|\theta^{2}+\theta-1\right|+|1-\theta|}{\theta^{2}}, \\
\gamma & =\frac{M}{2} \beta \eta, \\
a_{0} & =b_{0}=1, \quad d_{0}=\alpha+\gamma, b_{-1}=0, \\
a_{n+1} & =\frac{a_{n}}{1-a a_{n} d_{n}}, \\
b_{n+1} & =a_{n+1} \beta \eta c_{n}, \\
k_{n} & =\frac{|1+\theta|(\theta-1)^{2}+|1-\theta|}{\theta^{2}} b_{n}+\frac{M}{2} a_{n} \beta b_{n}^{2} \eta, \\
c_{n} & =\frac{M}{2} k_{n}^{2}+K|\theta| b_{n} k_{n}+\frac{M}{2}\left|\theta^{2}-1\right| b_{n}^{2}
\end{aligned}
$$

and

$$
d_{n+1}=\alpha b_{n+1}+\gamma a_{n+1} b_{n+1}^{2} .
$$

We suppose $\left(\mathcal{C}^{0}\right)$ :

(1) $\left\|F^{\prime}\left(x_{0}\right)^{-1}\left(F^{\prime}(x)-F^{\prime}(y)\right)\right\| \leq K\|x-y\|$ for each $x, y \in D$;

(2) $\left\|F^{\prime}\left(x_{0}\right)^{-1}\left(F^{\prime}(x)-F^{\prime}\left(x_{0}\right)\right)\right\| \leq K_{0}\left\|x-x_{0}\right\|$ for each $x \in D$;

(3) $\left\|F^{\prime}\left(x_{0}\right)^{-1} F\left(x_{0}\right)\right\| \leq \eta$.

Notice that the new conditions are given in affine invariant form and the condition on the second Fréchet-derivative has been dropped. The advantages of presenting results in affine invariant form instead of non-affine invariant form are well known $[2,4,12,17,20]$. If operator $F$ is twice Fréchet differentiable, then $(1)$ in $\left(\mathcal{C}^{0}\right)$ implies $(2)$ in $(\mathcal{C})$.

In order for us to compare the old approach with the new, let us rewrite the conditions $(\mathcal{C})$ in affine invariant form. We shall call these conditions again $(\mathcal{C})$.

$\left(\mathcal{C}_{1}\right)\left\|F^{\prime}\left(x_{0}\right)^{-1}\left(F^{\prime}(x)-F^{\prime}(y)\right)\right\| \leq K\|x-y\|$ for each $x$ and $y \in D$;

$\left(\mathcal{C}_{2}\right)\left\|F^{\prime}\left(x_{0}\right)^{-1} F^{\prime \prime}(x)\right\| \leq M$ for each $x \in D$;

$\left(\mathcal{C}_{4}\right)\left\|F^{\prime}\left(x_{0}\right)^{-1} F\left(x_{0}\right)\right\| \leq \eta$.

The parameters and sequences are defined as before but $\beta=1$. Then, we can certainly set $K=M$. Define parameters

$$
\begin{aligned}
& a^{0}=K \eta, \\
& \alpha^{0}=\alpha, \\
& \gamma^{0}=\frac{K}{2} \eta, \\
& a_{0}^{0}=b_{0}^{0}=1, d_{0}^{0}=\alpha^{0}+\gamma^{0}, b_{-1}^{0}=0,
\end{aligned}
$$




$$
\begin{aligned}
a_{n+1}^{0} & =\frac{1}{1-K_{0}\left(d_{n}^{0}+d_{n-1}^{0}+\cdots+d_{0}^{0}\right)}, \\
b_{n+1}^{0} & =a_{n+1}^{0} \eta c_{n}^{0}, \\
c_{n}^{0} & =K\left[\frac{\left(k_{n}^{0}\right)^{2}}{2}+|\theta| b_{n}^{0} k_{n}^{0}+\frac{\left|\theta^{2}-1\right|}{2}\left(b_{n}^{0}\right)^{2}\right], \\
k_{n}^{0} & =\frac{|\theta+1|(\theta-1)^{2}+|1-\theta|}{\theta^{2}} b_{n}^{0}+\frac{K}{2} a_{n}^{0}\left(b_{n}^{0}\right)^{2} \eta
\end{aligned}
$$

and

We have that

$$
d_{n+1}^{0}=\alpha^{0} b_{n+1}^{0}+\gamma^{0} a_{n+1}^{0}\left(b_{n+1}^{0}\right)^{2} .
$$

$$
K_{0} \leq K
$$

holds in general and $\frac{K}{K_{0}}$ can be arbitrarily large [2]-[4]. Notice that the center Lipschitz condition is not an additional condition to the Lipschitz condition, since in practice the computation of $K$ involves the computation of $K_{0}$ as a special case. We have by the definition of $a_{n+1}$ in turn that

$$
\begin{aligned}
a_{n+1} & =\frac{a_{n}}{1-K \eta a_{n} d_{n}} \\
& =\frac{a_{n}}{1-K \eta d_{n} \frac{a_{n-1}}{1-K \eta a_{n-1} d_{n-1}}} \\
& =\frac{a_{n}\left(1-K \eta a_{n-1} d_{n-1}\right)}{1-K \eta a_{n-1}\left(d_{n}+d_{n-1}\right)} \\
& =\frac{\frac{a_{n-1}}{1-K \eta a_{n-1} d_{n-1}}\left(1-K \eta a_{n-1} d_{n-1}\right)}{1-K \eta a_{n-1}\left(d_{n}+d_{n-1}\right)} \\
& =\frac{a_{n-1}}{1-K \eta a_{n-1}\left(d_{n}+d_{n-1}\right)} \\
& \vdots \\
& =\frac{a_{0}}{1-K \eta a_{n-1}\left(d_{n}+d_{n-1}+\cdots+d_{0}\right)} \\
& =\frac{1}{1-K \eta\left(d_{n}+d_{n-1}+\cdots+d_{0}\right)} .
\end{aligned}
$$

Hence, we deduce that

$$
a_{n+1}^{0} \leq a_{n+1} \text { for each } n=0,1,2, \ldots
$$

Moreover, strict inequality holds in (2.2) if $K_{0}<K$. Hence, using a simple inductive argument we also have that

$$
\begin{aligned}
b_{n+1}^{0} & \leq b_{n+1}, \\
c_{n}^{0} & \leq c_{n}, \\
k_{n}^{0} & \leq k_{n}
\end{aligned}
$$


and

$$
d_{n+1}^{0} \leq d_{n+1} .
$$

Lemma 2.1. Under the $\left(\mathcal{C}^{0}\right)$ conditions the following hold

$$
\begin{aligned}
\left\|F^{\prime}\left(x_{n}\right)^{-1} F^{\prime}\left(x_{0}\right)\right\| & \leq a_{n}^{0}, \\
\left\|F^{\prime}\left(x_{n}\right)^{-1} F\left(x_{n}\right)\right\| & \leq b_{n}^{0} \eta, \\
\left\|x_{n+1}-x_{n}\right\| & \leq d_{n}^{0} \eta, \\
\left\|x_{n+1}-y_{n}\right\| & \leq\left(d_{n}^{0}+2 k_{n-1}^{0}+\theta b_{n}^{0}\right) \eta .
\end{aligned}
$$

Moreover, under the $(\mathcal{C})$ conditions the following hold

$$
\begin{aligned}
\left\|F^{\prime}\left(x_{n}\right)^{-1} F^{\prime}\left(x_{0}\right)\right\| & \leq a_{n}^{0} \leq a_{n}, \\
\left\|F^{\prime}\left(x_{n}\right)^{-1} F\left(x_{n}\right)\right\| & \leq b_{n}^{0} \eta \leq b_{n} \eta, \\
\left\|x_{n+1}-x_{n}\right\| & \leq d_{n}^{0} \eta \leq d_{n} \eta \\
\left\|x_{n+1}-y_{n}\right\| & \leq\left(d_{n}^{0}+2 k_{n-1}^{0}+\theta b_{n}^{0}\right) \eta \leq\left(d_{n}+2 k_{n-1}+\theta b_{n}\right) \eta .
\end{aligned}
$$

Proof. It follows from the proof of Lemma 1 in [7] by simply noticing: the expressions involving

(i) the second Fréchet-derivative

$$
\int_{0}^{1} F^{\prime \prime}\left(x_{n}+t\left(y_{n}-x_{n}\right)\right)(1-t)\left(y_{n}-x_{n}\right)^{2} d t
$$

and

$$
\int_{0}^{1} F^{\prime \prime}\left(y_{n}+t\left(x_{n+1}-y_{n}\right)\right)(1-t)\left(x_{n+1}-y_{n}\right)^{2} d t
$$

are not needed and can be replaced, respectively, by

$$
\int_{0}^{1}\left[F^{\prime}\left(y_{n}+t\left(x_{n}-y_{n}\right)-F^{\prime}\left(x_{n}\right)\right]\left(y_{n}-x_{n}\right) d t\right.
$$

and

$$
\int_{0}^{1}\left[F^{\prime}\left(y_{n}+t\left(x_{n+1}-y_{n}\right)-F^{\prime}\left(y_{n}\right)\right]\left(x_{n+1}-y_{n}\right) d t .\right.
$$

Hence, condition $(2)$ in $(\mathcal{C})$ is not needed and can be replaced by condition $(1)$ in $\left(\mathcal{C}^{0}\right)$ to produce the same bounds as in [7] (for $K=M$ ) (see also the proof of Theorem 3.2 that follows).

(ii) The computation of the upper bounds on $\left\|F^{\prime}\left(x_{n}\right)^{-1} F^{\prime}\left(x_{0}\right)\right\|$ in [7] uses condition $(1)$ in $(\mathcal{C})$ and the estimate

$$
\left\|F^{\prime}\left(x_{n}\right)^{-1}\left(F^{\prime}\left(x_{n}\right)-F^{\prime}\left(x_{n+1}\right)\right)\right\| \leq\left\|F^{\prime}\left(x_{n}\right)^{-1} F^{\prime}\left(x_{0}\right)\right\| K\left\|x_{n}-x_{n+1}\right\|
$$

to arrive at

$$
\left\|F^{\prime}\left(x_{n}\right)^{-1} F^{\prime}\left(x_{0}\right)\right\| \leq a_{n+1},
$$

whereas we use $(2)$ in $\left(\mathcal{C}^{0}\right)$ and estimate

$$
\left\|F^{\prime}\left(x_{0}\right)^{-1}\left(F^{\prime}\left(x_{n}\right)-F^{\prime}\left(x_{n+1}\right)\right)\right\| \leq K_{0}\left\|x_{n+1}-x_{n}\right\|
$$




$$
\begin{aligned}
& \leq K_{0}\left(\left\|x_{n+1}-x_{n}\right\|+\cdots+\left\|x_{1}-x_{0}\right\|\right) \\
& \leq K_{0}\left(d_{n}^{0}+d_{n-1}^{0}+\cdots+d_{0}^{0}\right)
\end{aligned}
$$

to arrive at the estimate

$$
\left\|F^{\prime}\left(x_{n}\right)^{-1} F^{\prime}\left(x_{0}\right)\right\| \leq a_{n+1}^{0},
$$

which is more precise (see also (2.2)).

Lemma 2.2. Suppose that

$$
a_{1}^{0} b_{1}^{0}<1
$$

Then, sequence $\left\{p_{n}^{0}\right\}$ defined by $p_{n}^{0}=a_{n}^{0} b_{n}^{0}$ is decreasingly convergent to 0 such that

and

$$
p_{n+1}^{0} \leq \xi_{1}^{2^{n+1}} \frac{1}{\xi_{1}}, \xi_{1}:=a_{1}^{0} b_{1}^{0}
$$

Moreover, if

$$
d_{n}^{0} \leq\left(\alpha^{0}+\gamma^{0}\right) \xi_{1}^{2^{n}} \frac{1}{\xi_{1}} .
$$

$$
a_{1} b_{1}<1,
$$

then, sequence $\left\{p_{n}\right\}$ defined by $p_{n}=a_{n} b_{n}$ is also decreasingly convergent to 0 such that

and

$$
\begin{gathered}
p_{n+1}^{0} \leq p_{n+1} \leq \xi^{2^{n+1}} \frac{1}{\xi}, \xi=a_{1} b_{1}, \\
d_{n}^{0} \leq d_{n} \leq(\alpha+\gamma) \xi^{2^{n}} \frac{1}{\xi}
\end{gathered}
$$

$$
\xi_{1} \leq \xi
$$

Proof. It follows from the proof of Lemma 3 in [7] by simply using $\left\{p_{n}^{0}\right\}, a_{1}^{0}$, $b_{1}^{0}, \xi_{1}$ instead of $\left\{p_{n}\right\}, a_{1}, b_{1}, \xi$, respectively.

Next, we present the main semilocal convergence result for the third order method (1.2) under the $\left(\mathcal{C}^{0}\right)$ conditions, $(2.9)$ and the convergence criterion

$$
a(\alpha+\gamma)<1 \text {. }
$$

The proof follows from the proof of Theorem 5 in [7] (with the exception of the uniqueness of the solution part) by simply replacing the $(\mathcal{C})$ conditions and (2.10) by the $\left(\mathcal{C}^{0}\right)$ conditions and (2.9) respectively.

Theorem 2.3. Suppose that conditions $\left(\mathcal{C}^{0}\right)$, (2.9) and (2.11) hold. Moreover, suppose that

$$
U_{0}^{0}=\bar{U}\left(x_{0}, r^{0} \eta\right) \subset D,
$$

where

$$
r_{0}=\sum_{n=0}^{\infty} d_{n}^{0}
$$


Then, sequences $\left\{x_{n}\right\}$ generated by the third order method (1.2) is well defined, remains in $U_{0}^{0}$ for each $n=0,1,2, \ldots$ and converges to a unique solution $x^{*}$ of equation $F(x)=0$ in $U\left(x_{0}, \frac{2}{K_{0}}-r_{0} \eta\right) \bigcap D$. Moreover, the following estimates hold

$$
\left\|x_{n+1}-x^{*}\right\| \leq \sum_{k=n+1}^{\infty} d_{k}^{0} \eta \leq \frac{\alpha+\gamma}{\xi_{1}} \eta \sum_{k=n+1}^{\infty} \xi_{1}^{2^{k}} .
$$

Proof. As already noted above, we only need to show the uniqueness part. Let $y^{*} \in U\left(x_{0}, \frac{2}{K_{0}}-r_{0} \eta\right)$ be such that $F\left(y^{*}\right)=0$. Define $Q=\int_{0}^{1} F^{\prime}\left(x^{*}+t\left(y^{*}-\right.\right.$ $\left.\left.x^{*}\right)\right) d t$. Using condition $(2)$ in $\left(\mathcal{C}^{0}\right)$ we get in turn that

$$
\begin{aligned}
\left\|F^{\prime}\left(x_{0}\right)^{-1}\left(F^{\prime}\left(x_{0}\right)-Q\right)\right\| & \leq K_{0} \int_{0}^{1}\left\|x^{*}+t\left(y^{*}-x^{*}\right)-x_{0}\right\| d t \\
& \leq K_{0} \int_{0}^{1}\left[(1-t)\left\|x^{*}-x_{0}\right\|+t\left\|y^{*}-x_{0}\right\|\right] d t \\
& <\frac{K_{0}}{2}\left[r_{0} \eta+\frac{2}{K_{0}}-r_{0} \eta\right]=1 .
\end{aligned}
$$

It follows from (2.15) and the Banach lemma on invertible operators $[2,4,12$, $17,20]$ that $Q^{-1} \in L(Y, X)$. Then, using the identity

$$
0=F\left(x^{*}\right)-F\left(y^{*}\right)=Q\left(x^{*}-y^{*}\right),
$$

we deduce that $x^{*}=y^{*}$.

Remark 2.4. If $K_{0}=K$, and operator $F$ is twice Fréchet differentiable, then Lemma 2.1, Lemma 2.2 and Theorem 2.3 reduce to Lemma 1, Lemma 3 and Theorem 5 in [7], respectively. Otherwise, i.e., if $K_{0}<K$ or if the twice Fréchet differentiability of operator $F$ is not assumed, then our results constitute an improvement. It is worth noticing that if $K_{0}<K$, then (2.10) implies (2.9) (but not necessarily vice versa) and $\xi_{1}<\xi$.

\section{Semilocal convergence II}

We need to introduce some scalar sequences that shall be shown to be majorizing for the third order methods (1.2) in Theorem 3.2.

Let $K_{0}>0, K>0, \eta>0$ and $\theta \in \mathbb{R}-\{0\}$. Set $t_{0}=0$ and $s_{0}=|\theta| \eta$. Define polynomials $f$ and $g$ by

$$
f(t)\left(\frac{K|\theta|}{2}+K_{0}\right) t^{3}+\frac{|\theta|}{2} K t^{2}+K\left(\frac{\left|\theta^{2}-1\right|}{2|\theta|}-|\theta|\right) t-\frac{K}{2} \frac{\left|\theta^{2}-1\right|}{|\theta|}
$$

and

$$
\begin{aligned}
g(t)= & K_{0} t^{4}+\frac{K}{2 \theta^{2}}\left[1+|1-\theta|\left(1+\left|1-\theta^{2}\right|\right)\right] t^{3}+\frac{K}{2 \theta^{2}}\left[|1-\theta|\left(1+\left|1-\theta^{2}\right|\right)-1\right] t^{2} \\
& +\frac{K}{\theta^{2}}|1-\theta|\left(1+\left|1-\theta^{2}\right|\right)\left(\frac{\left|\theta^{2}-1\right|}{2 \theta^{2}}-1\right) t-\frac{K}{2 \theta^{4}}|1-\theta|\left|1-\theta^{2}\right|\left(1+\left|1-\theta^{2}\right|\right) .
\end{aligned}
$$


We have $f(0)=-\frac{K}{2} \frac{\left|\theta^{2}-1\right|}{|\theta|}<0$ for $\theta \neq \pm 1$ and $f(1)=K_{0}>0$ for $K_{0} \neq 0$. It follows from the intermediate value theorem that polynomial $f$ has roots in $(0,1)$. Denote by $\delta_{f}$ the smallest root of $f$ in $(0,1)$. Similarly, we have $g(0)=-\frac{K}{2 \theta^{4}}|1-\theta|\left|\theta^{2}-1\right|\left(1+\left|1-\theta^{2}\right|\right)<0$ for $\theta \neq \pm 1$ and $g(1)=K_{0}+\frac{K}{2 \theta^{2}}>0$. Denote by $\delta_{g}$ the smallest root of $g$ in $(0,1)$. Set

$$
\delta=\min \left\{\delta_{f}, \delta_{g}\right\}
$$

Moreover, suppose that $\delta$ satisfies

$$
\begin{gathered}
\left|\frac{1-\theta}{\theta^{3}}\right|\left(1+\left|1-\theta^{2}\right|\right)+\frac{K \eta}{2 \theta} \leq \delta, \\
0<\frac{K|\theta|}{1-K_{0}(1+\delta) s_{0}}\left[\frac{\left|\theta^{2}-1\right|}{2 \theta^{2}}+\frac{\delta^{2}}{2}+\delta\right]\left(s_{0}-t_{0}\right) \leq \delta
\end{gathered}
$$

and

$$
\begin{array}{r}
0<\frac{K}{\theta^{2}\left(1-K_{0}(1+\delta) s_{0}\right)}\left\{|1-\theta|\left(1+\left|1-\theta^{2}\right|\right)\right. \\
\left.\quad\left[\frac{\left|\theta^{2}-1\right|}{2 \theta^{2}}+\frac{\delta^{2}}{2}+\delta\right]+\frac{\delta^{2}}{2}\right\}\left(s_{0}-t_{0}\right) \leq \delta^{2}
\end{array}
$$

We shall assume from now on that $\delta$ satisfies conditions (3.3)-(3.6). These conditions shall be referred to as the $(\triangle)$ conditions. Moreover, define scalar sequences $\left\{t_{n}\right\},\left\{s_{n}\right\}$ by

$$
\begin{aligned}
& t_{0}=0, s_{0}=t_{0}+\theta \eta \\
& t_{1}=s_{0}+\left[\frac{|1-\theta|}{\left|\theta^{3}\right|}\left(1+\left|1-\theta^{2}\right|\right)+\frac{\left(s_{0}-t_{0}\right) K}{2 \theta^{2}}\right]\left(s_{0}-t_{0}\right)
\end{aligned}
$$

for each $n=0,1,2, \ldots$.

$$
s_{n+1}=t_{n+1}+\frac{K|\theta|}{1-K_{0} t_{n+1}}
$$

$$
\begin{aligned}
& {\left[\frac{\left|1-\theta^{2}\right|}{2 \theta^{2}}\left(s_{n}-t_{n}\right)^{2}+\frac{\left(t_{n+1}-s_{n}\right)^{2}}{2}+\left(s_{n}-t_{n}\right)\left(t_{n+1}-s_{n}\right)\right], } \\
t_{n+2}= & s_{n+1}+\frac{K}{\theta^{2}\left(1-K_{0} t_{n+1}\right)}\left\{|1-\theta|\left(1+\left|1-\theta^{2}\right|\right)\right. \\
& {\left[\frac{\left|1-\theta^{2}\right|}{2 \theta^{2}}\left(s_{n}-t_{n}\right)^{2}+\frac{\left(t_{n+1}-s_{n}\right)^{2}}{2}+\left(s_{n}-t_{n}\right)\left(t_{n+1}-s_{n}\right)\right] } \\
+ & \left.\frac{1}{2}\left(s_{n+1}-t_{n+1}\right)^{2}\right\}
\end{aligned}
$$

Then, we can show the following auxiliary result for majorizing sequences $\left\{t_{n}\right\}$, $\left\{s_{n}\right\}$ under the $(\triangle)$ conditions. 
Lemma 3.1. Suppose that the $(\triangle)$ conditions hold. Then, sequence $\left\{t_{n}\right\},\left\{s_{n}\right\}$ defined by (3.7) and (3.8) are increasingly convergent to their unique least upper bound denoted by $t^{*}$ which satisfies

$$
\theta \eta \leq t^{*} \leq t^{* *}:=\frac{\theta \eta}{1-\delta}
$$

Moreover, the following estimates hold for each $n=0,1,2, \ldots$.

$$
0<s_{n}-t_{n} \leq \delta^{n} \theta \eta
$$

and

$$
0<t_{n+1}-s_{n} \leq \delta^{n+1} \theta \eta
$$

Proof. We shall show estimates (3.10) and (3.11) using induction. If $n=0$, (3.10) holds by the definition of $t_{0}$ and $s_{0}$, whereas (3.11) holds by (3.4). We then have that

$$
t_{1} \leq s_{0}+\delta s_{0}=(1+\delta) s_{0}=\frac{1-\delta^{2}}{1-\delta} s_{0}<t^{* *}
$$

If $n=1$, estimates (3.10) and (3.11) hold by (3.5), (3.6), (3.12) and (3.10), (3.11) for $n=0$. Suppose that (3.10) and (3.11) hold for all $m \leq n$. Then, we have that

$$
\begin{aligned}
t_{m+1} \leq & s_{m}+\delta^{m+1}\left(s_{0}-t_{0}\right) \leq t_{m}+\delta^{m}\left(s_{0}-t_{0}\right) \\
& +\delta^{m+1}\left(s_{0}-t_{0}\right) \leq \cdots \leq t_{0}+\left(s_{0}-t_{0}\right)+\delta\left(s_{0}-t_{0}\right) \\
& +\cdots+\delta^{m+1}\left(s_{0}-t_{0}\right)=\frac{1-\delta^{m+2}}{1-\delta}\left(s_{0}-t_{0}\right)<t^{* *}
\end{aligned}
$$

Next, we shall show (3.10) for $m+1$ replacing $n$. We have by the induction hypotheses and (3.13) that

$$
\begin{aligned}
s_{m+1}-t_{m+1} \leq & \frac{K|\theta|}{1-K_{0} \frac{1-\delta^{m+2}}{1-\delta}} \\
& {\left[\frac{\left|\theta^{2}-1\right|}{\theta^{2}}\left(\delta^{m}\left(s_{0}-t_{0}\right)\right)^{2}+\frac{\left(\delta^{m}\left(s_{0}-t_{0}\right)\right)^{2}}{2}+\delta^{2 m+1}\left(s_{0}-t_{0}\right)^{2}\right] }
\end{aligned}
$$

must be smaller or equal to $\delta^{m+1}\left(s_{0}-t_{0}\right)$, or

$$
\frac{K|\theta|}{1-K_{0} \frac{1-\delta^{m+2}}{1-\delta}}\left[\frac{\left|\theta^{2}-1\right|}{\theta^{2}} \delta^{m}+\frac{\delta^{m+2}}{2}+\delta^{m+1}\right]\left(s_{0}-t_{0}\right) \leq \delta .
$$

Estimate (3.14) motivates us to define recurrent polynomials $f_{m}$ on $(0,1)$ by

$$
\begin{aligned}
f_{m}(t)= & K\left[\frac{|\theta|}{2} t^{m+2}+|\theta| t^{m+1}+\frac{\left|\theta^{2}-1\right|}{2|\theta|} t^{m}\right]\left(s_{0}-t_{0}\right) \\
& +K_{0} t\left(1+t+\cdots+t^{m+1}\right)\left(s_{0}-t_{0}\right)-t .
\end{aligned}
$$


We need a relationship between two consecutive polynomials $f_{m}$. Using (3.15) and (3.1) by direct algebraic manipulation we get that

$$
f_{m+1}(t)=f_{m}(t)+f(t) t^{m-1}\left(s_{0}-t_{0}\right) .
$$

Evidently, condition (3.14) is satisfied, if

$$
f_{m}(\delta) \leq 0 \text {. }
$$

We also have from (3.17) that

$$
f_{m+1}(\delta) \leq f_{m}(\delta)
$$

since $f(\delta) \leq 0$. It then, follows from (3.17) and (3.18) that (3.17) holds, if

$$
f_{0}(\delta) \leq 0,
$$

which is true by (3.5). Hence, we showed (3.10) for $m+1$ replacing $n$. Next, we shall show (3.11) for $m+1$ replacing $n$. We have in turn that

$$
\begin{aligned}
s_{m+2}-s_{m+1} \leq & \frac{K}{\theta^{2}\left(1-K_{0} \frac{1-\delta^{m+2}}{1-\delta}\right)}\left\{|1-\theta|\left(1+\left|\theta^{2}-1\right|\right)\right. \\
& {\left[\frac{\left|\theta^{2}-1\right|}{2 \theta^{2}}\left(\delta^{m}\left(s_{0}-t_{0}\right)\right)^{2}+\frac{\left(\delta^{m+1}\left(s_{0}-t_{0}\right)\right)^{2}}{2}+\delta^{2 m+1}\left(s_{0}-t_{0}\right)^{2}\right] } \\
& \left.+\left(\delta^{m+1}\left(s_{0}-t_{0}\right)\right)^{2}\right\}
\end{aligned}
$$

must be smaller or equal to $\delta^{m+2}\left(s_{0}-t_{0}\right)$. As in the preceding case we are motivated to define polynomials $g_{m}$ on $[0,1]$ by

$$
\begin{aligned}
g_{m}(t)= & K\left\{\frac{|1-\theta|\left(1+\left|\theta^{2}-1\right|\right)}{\theta^{2}}\left[\frac{\left|\theta^{2}-1\right|}{\theta^{2}} t^{m}+\frac{t^{m+2}}{2}+t^{m+1}\right]+\frac{t^{m+2}}{2 \theta^{2}}\right\} \\
& \times\left(s_{0}-t_{0}\right)+t^{2} K_{0}\left(1+t+\cdots+t^{m+1}\right)\left(s_{0}-t_{0}\right)-t^{2}
\end{aligned}
$$

Using (3.20) and (3.2) by direct algebraic manipulation we get that

$$
g_{m+1}(t)=g_{m}(t)+g(t) t^{m}\left(s_{0}-t_{0}\right) .
$$

Condition (3.11) is satisfied, if

$$
g_{m}(\delta) \leq 0 .
$$

We also have from $(3.21)$ and $(\triangle)$ that

$$
g_{m+1}(\delta) \leq g_{m}(\delta),
$$

since $g(\delta) \leq 0$. Hence, (3.22) is satisfied, if

$$
g_{0}(\delta) \leq 0,
$$

which is true by (3.6). The induction for (3.11) is completed. It then, follows that

$$
t_{m+2} \leq \frac{1-\delta^{m+3}}{1-\delta} s_{0}<t^{* *}
$$

Hence, sequences $\left\{t_{n}\right\},\left\{s_{n}\right\}$ are increasing, bounded above by $t^{* *}$ and as such they converge to their unique least upper bound $t^{*}$ which satisfies (3.9). 
We can show the main semilocal convergence result for the third order method (1.2) under the $\left(\mathcal{C}^{0}\right)$ and $(\triangle)$ conditions using $\left\{t_{n}\right\}$ and $\left\{s_{n}\right\}$ as majorizing sequences.

Theorem 3.2. Suppose that

$$
\bar{U}\left(x_{0}, t^{*}\right) \subset D
$$

the $\left(\mathcal{C}^{0}\right)$ and $(\triangle)$ conditions hold. Then, sequences $\left\{x_{n}\right\},\left\{y_{n}\right\}$ generated by the third order method (1.2) are well defined, remain in $\bar{U}\left(x_{0}, t^{*}\right)$ for each $n=0,1,2, \ldots$ and converge to a unique solution $x^{*}$ of equation $F(x)=0$ in $\bar{U}\left(x_{0}, t^{*}\right) \cap D$. Moreover the following estimates hold for each $n=0,1,2, \ldots$.

$$
\begin{aligned}
\left\|y_{n}-x_{n}\right\| & \leq s_{n}-t_{n}, \\
\left\|x_{n+1}-y_{n}\right\| & \leq t_{n+1}-s_{n}, \\
\left\|x_{n+1}-x_{n}\right\| & \leq t_{n+1}-t_{n},
\end{aligned}
$$

and

$$
\left\|x_{n}-x^{*}\right\| \leq t^{*}-t_{n}
$$

Furthermore, if there exists $R>t^{*}$ such that

$$
K_{0}\left(t^{*}+R\right)<2,
$$

then, the point $x^{*}$ is the only solution of equation $F(x)=0$ in $U\left(x_{0}, R\right)$.

Proof. We shall first show (3.27) and (3.28) using induction. We have by (1.2) and (3.7) that

$$
\left\|y_{0}-x_{0}\right\|=|\theta||| F^{\prime}\left(x_{0}\right)^{-1} F\left(x_{0}\right) \| \leq|\theta| \eta=s_{0}=s_{0}-t_{0} .
$$

Hence, (3.27) holds for $n=0$. It follows from the first substep of (1.2) that

$$
\begin{aligned}
F\left(y_{0}\right) & =F\left(y_{0}\right)-\theta F\left(x_{0}\right)-F^{\prime}\left(x_{0}\right)\left(y_{0}-x_{0}\right) \\
2) & =(1-\theta) F\left(x_{0}\right)+\int_{0}^{1}\left[F^{\prime}\left(x_{0}+t\left(y_{0}-x_{0}\right)\right)-F^{\prime}\left(x_{0}\right)\right]\left(y_{0}-x_{0}\right) d t .
\end{aligned}
$$

Composing (3.32) by $F^{\prime}\left(x_{0}\right)^{-1}$ and using (2), (3) in $\left(\mathcal{C}^{0}\right)$ and (3.7)

$$
\begin{aligned}
\left\|F^{\prime}\left(x_{0}\right)^{-1} F\left(y_{0}\right)\right\| \leq & |1-\theta|\|\| F^{\prime}\left(x_{0}\right)^{-1} F\left(x_{0}\right) \| \\
& +\| \int_{0}^{1}\left[F^{\prime}\left(x_{0}+t\left(y_{0}-x_{0}\right)\right)-F^{\prime}\left(x_{0}\right)\right]\left(y_{0}-x_{0}\right) d t \\
\leq & \frac{|1-\theta|}{|\theta|}\left(s_{0}-t_{0}\right)+\frac{K_{0}}{2}\left\|y_{0}-x_{0}\right\|^{2} \\
\leq & \left(\frac{|1-\theta|}{|\theta|}+\frac{K_{0}}{2}\left(s_{0}-t_{0}\right)\right)\left(s_{0}-t_{0}\right) .
\end{aligned}
$$

Subtracting the first from the second substep in (1.2) we get that

$$
x_{1}-y_{0}=-\frac{(\theta+1)(\theta-1)^{2}}{\theta^{2}} F^{\prime}\left(x_{0}\right)^{-1} F\left(x_{0}\right)-\frac{1}{\theta^{2}} F^{\prime}\left(x_{0}\right)^{-1} F\left(y_{0}\right) \text {. }
$$


Hence, using (3.33) and (3.34), we get that

$$
\begin{aligned}
\left\|x_{1}-y_{0}\right\| & =\frac{|\theta+1||\theta-1|^{2}}{\theta^{2}}\left\|F^{\prime}\left(x_{0}\right)^{-1} F\left(x_{0}\right)\right\|+\frac{1}{\theta^{2}}\left\|F^{\prime}\left(x_{0}\right)^{-1} F\left(y_{0}\right)\right\| \\
& \leq \frac{|\theta+1||\theta-1|^{2}}{\theta^{2}}\left(s_{0}-t_{0}\right)+\frac{1}{\theta^{2}}\left(\frac{|1-\theta|}{|\theta|}+\frac{K}{2}\left(s_{0}-t_{0}\right)\right)\left(s_{0}-t_{0}\right) \\
& =t_{1}-s_{0},
\end{aligned}
$$

which shows (3.28) for $n=0$. Then, (3.29) holds for $n=0$, since

$$
\left\|x_{1}-x_{0}\right\| \leq\left\|x_{1}-y_{0}\right\|+\left\|y_{0}-x_{0}\right\| \leq t_{1}-s_{0}+s_{0}-t_{0}=t_{1}-t_{0} \leq t^{*} .
$$

Then, we have $x_{1} \in \bar{U}\left(x_{0}, t^{*}\right)$. Notice that $K_{0} t^{*}<1$ from the proof of Lemma 3.1. Let us suppose $x \in \bar{U}\left(x_{0}, t^{*}\right)$. Then, using $(2)$ in $\left(\mathcal{C}^{0}\right)$ we have that

$$
\left\|F^{\prime}\left(x_{0}\right)^{-1}\left(F^{\prime}(x)-F^{\prime}\left(x_{0}\right)\right)\right\| \leq K_{0}\left\|x-x_{0}\right\| \leq K_{0} t^{*}<1 .
$$

It follows from (3.36) and the Banach lemma that $F^{\prime}(x)^{-1} \in L(Y, X)$ and

$$
\left\|F^{\prime}\left(x_{1}\right)^{-1} F^{\prime}\left(x_{0}\right)\right\| \leq \frac{1}{1-K_{0}\left\|x_{1}-x_{0}\right\|} \leq \frac{1}{1-K_{0} t_{1}} .
$$

Suppose that (3.27)-(3.29) hold for all $m \leq n$ and $x_{m} \in \bar{U}\left(x_{0}, t^{*}\right)$. Using the first step in (1.2) we get that

$$
\begin{aligned}
F\left(y_{m}\right) & =F\left(y_{m}\right)-\theta F\left(x_{m}\right)-F^{\prime}\left(x_{m}\right)\left(y_{m}-x_{m}\right) \\
(3.38) & =(1-\theta) F\left(x_{m}\right)+\int_{0}^{1}\left[F^{\prime}\left(x_{m}+t\left(y_{m}-x_{m}\right)\right)-F^{\prime}\left(x_{m}\right)\right]\left(y_{m}-x_{m}\right) d t .
\end{aligned}
$$

Subtracting the first step in (1.2) from the second step to obtain

$$
F^{\prime}\left(x_{m}\right)\left(x_{m+1}-y_{m}\right)=\frac{\theta^{3}-\theta^{2}-\theta+1}{\theta^{2}} F\left(x_{m}\right)-\frac{1}{\theta^{2}} F\left(y_{m}\right) .
$$

We also have by (3.38) that

$$
\begin{aligned}
F\left(x_{m+1}\right)= & F^{\prime}\left(x_{m}\right)\left(x_{m+1}-y_{m}\right)+F\left(y_{m}\right)+\left[F^{\prime}\left(y_{m}\right)-F^{\prime}\left(x_{m}\right)\right]\left(x_{m+1}-y_{m}\right) \\
& +F\left(x_{m+1}\right)-F\left(y_{m}\right)-F^{\prime}\left(y_{m}\right)\left(x_{m+1}-y_{m}\right) \\
= & \frac{1-\theta}{\theta^{2}} F\left(x_{m}\right)-\frac{1}{\theta^{2}} F\left(y_{m}\right) \\
& +\int_{0}^{1}\left[F^{\prime}\left(x_{m}+t\left(y_{m}-x_{m}\right)\right)-F^{\prime}\left(x_{m}\right)\right]\left(y_{m}-x_{m}\right) d t \\
& +\int_{0}^{1}\left[F^{\prime}\left(y_{m}+t\left(x_{m+1}-y_{m}\right)\right)-F^{\prime}\left(y_{m}\right)\right]\left(x_{m+1}-y_{m}\right) d t \\
& +\left[F^{\prime}\left(y_{m}\right)-F^{\prime}\left(x_{m}\right)\right]\left(x_{m+1}-y_{m}\right) .
\end{aligned}
$$

Hence, we get by (3.40) that

$$
\left\|F^{\prime}\left(x_{0}\right)^{-1} F\left(x_{m+1}\right)\right\| \leq K\left[\frac{\left|\theta^{2}-1\right|}{2 \theta^{2}}\left\|y_{m}-x_{m}\right\|^{2}\right.
$$




$$
\begin{aligned}
& \left.+\frac{\left\|x_{m+1}-y_{m}\right\|^{2}}{2}+\left\|y_{m}-x_{m}\right\|\left\|x_{m+1}-y_{m}\right\|\right] \\
\leq & K\left[\frac{\left|\theta^{2}-1\right|}{2 \theta^{2}}\left(s_{m}-t_{m}\right)^{2}\right. \\
& \left.+\frac{\left(t_{m+1}-s_{m}\right)^{2}}{2}+\left(s_{m}-t_{m}\right)\left(t_{m+1}-s_{m}\right)\right] .
\end{aligned}
$$

Then, we get that

$$
\begin{aligned}
\left\|y_{m+1}-x_{m+1}\right\| \leq & \left\|F^{\prime}\left(x_{m+1}\right)^{-1} F^{\prime}\left(x_{0}\right)\right\|\left\|F^{\prime}\left(x_{0}\right)^{-1} F\left(x_{m+1}\right)\right\| \\
\leq & \frac{K}{1-K_{0} t_{m+1}}\left[\frac{\left|\theta^{2}-1\right|}{2 \theta^{2}}\left(s_{m}-t_{m}\right)^{2}\right. \\
& \left.+\frac{\left(t_{m+1}-s_{m}\right)^{2}}{2}+\left(s_{m}-t_{m}\right)\left(t_{m+1}-s_{m}\right)\right] \\
= & s_{m+1}-t_{m+1},
\end{aligned}
$$

where, we used (3.37) for $x=x_{m+1}$ and

$\left\|x_{m+1}-x_{0}\right\| \leq\left\|x_{m+1}-x_{m}\right\|+\cdots+\left\|x_{1}-x_{0}\right\| \leq t_{m+1}-t_{m}+\cdots+t_{1}-t_{0}=t_{m+1}$.

Hence, we showed (3.27). Then, we have by (3.39) that

(3.42) $x_{m+1}-y_{m}=\frac{\theta^{3}-\theta^{2}-\theta+1}{\theta^{2}} F^{\prime}\left(x_{m}\right)^{-1} F\left(x_{m}\right)-\frac{1}{\theta^{2}} F^{\prime}\left(x_{m}\right)^{-1} F\left(y_{m}\right)$.

It follows from (3.42) that

$$
\begin{aligned}
\left\|x_{m+2}-y_{m+1}\right\| \leq & \frac{K}{1-K_{0} t_{m+1}}\left[\frac{|1+\theta|(\theta-1)^{2}}{\theta^{2}}\left\|F^{\prime}\left(x_{0}\right)^{-1} F\left(x_{m+1}\right)\right\|\right. \\
& \left.+\frac{1}{\theta^{2}}\left\|F^{\prime}\left(x_{0}\right)^{-1} F\left(y_{m+1}\right)\right\|\right] \\
\leq & \frac{K}{\theta^{2}\left(1-K_{0} t_{m+1}\right)}\left[| 1 + \theta | ( \theta - 1 ) ^ { 2 } \left(\frac{\theta^{2}-1 \mid}{2 \theta^{2}}\left(s_{m}-t_{m}\right)^{2}\right.\right. \\
& \left.+\frac{\left(t_{m+1}-s_{m}\right)^{2}}{2}+\left(s_{m}-t_{m}\right)\left(t_{m+1}-s_{m}\right)\right) \\
& +|1-\theta|\left(\frac{\left|\theta^{2}-1\right|}{2 \theta^{2}}\left(s_{m}-t_{m}\right)^{2}\right. \\
& \left.\left.+\frac{\left(t_{m+1}-s_{m}\right)^{2}}{2}+\left(s_{m}-t_{m}\right)\left(t_{m+1}-s_{m}\right)\right)\right) \\
& \left.+\frac{\left(s_{m+1}-t_{m+1}\right)^{2}}{2}\right] \\
= & t_{m+2}-s_{m+1} .
\end{aligned}
$$

Hence, we showed (3.28). Then, we have that

$$
\begin{aligned}
\left\|x_{m+2}-x_{m+1}\right\| & \leq\left\|x_{m+2}-y_{m+1}\right\|+\left\|y_{m+1}-x_{m+1}\right\| \\
& \leq t_{m+2}-s_{m+1}+s_{m+1}-t_{m+1}
\end{aligned}
$$




$$
=t_{m+2}-t_{m+1},
$$

which shows (3.29). We also have that

$$
\begin{aligned}
\left\|x_{m+2}-x_{0}\right\| & \leq\left\|x_{m+2}-x_{m+1}\right\|+\left\|x_{m+1}-x_{m}\right\|+\cdots+\left\|x_{1}-x_{0}\right\| \\
& \leq t_{m+2}-t_{m+1}+t_{m+1}-t_{m}+\cdots+t_{1}-t_{0} \\
& =t_{m+2}<t^{*}
\end{aligned}
$$

Hence, we get $x_{m+2} \in \bar{U}\left(x_{0}, t^{*}\right)$.

We showed in Lemma 3.1 that sequences $\left\{t_{n}\right\},\left\{s_{n}\right\}$ are complete. Hence, it follows from (3.27)-(3.29) that sequences $\left\{x_{n}\right\},\left\{y_{n}\right\}$ are complete in a Banach space $X$ and as such they converge to some $x^{*} \in \bar{U}\left(x_{0}, t^{*}\right)$ (since $\bar{U}\left(x_{0}, t^{*}\right)$ is a closed set.) By letting $m \rightarrow \infty$ in (3.41), we obtain $F\left(x^{*}\right)=0$. Estimate (3.30) follows from (3.29) by using standard majorization techniques $[2,4,12,17,20$, 21]. Let us show uniqueness, first in $\bar{U}\left(x_{0}, t^{*}\right) \cap D$. Let $y^{*} \in \bar{U}\left(x_{0}, t^{*}\right)$ be such that $F\left(y^{*}\right)=0$. Set $Q=\int_{0}^{1} F^{\prime}\left(x^{*}+t\left(y^{*}-x^{*}\right)\right) d t$. Then, using $(2)$ in $\left(\mathcal{C}^{0}\right)$ we get that

$$
\begin{aligned}
\left\|F^{\prime}\left(x_{0}\right)^{-1}\left(F^{\prime}\left(x_{0}\right)-Q\right)\right\| & \leq K_{0} \int_{0}^{1}\left[(1-t)\left\|x^{*}+t\left(y^{*}-x^{*}\right)-x_{0}\right\| d t\right. \\
& \leq K_{0} \int_{0}^{1}\left[(1-t)\left\|x^{*}-x_{0}\right\|+t \| y^{*}-x^{*}\right)-x_{0} \| d t \\
& \leq K_{0} t^{*}<1 .
\end{aligned}
$$

It follows that $Q^{-1}$ exists. Then, from the identity $0=F\left(x^{*}\right)-F\left(y^{*}\right)=$ $Q\left(x^{*}-y^{*}\right)$ we deduce that $x^{*}=y^{*}$. Similarly, if $F\left(y^{*}\right)=0$ and $y^{*} \in U\left(x_{0}, R\right)$, we have that

$$
\left\|F^{\prime}\left(x_{0}\right)^{-1}\left(F^{\prime}\left(x_{0}\right)-Q\right)\right\| \leq \frac{K_{0}}{2}\left(R+t^{*}\right)<1,
$$

by (3.31). Hence, again we deduce that $x^{*}=y^{*}$.

Remark 3.3. (a) It follows from the proof of Theorem 3.2 that sequences $\left\{\bar{t}_{n}\right\},\left\{\bar{s}_{n}\right\}$ defined by

$$
\begin{aligned}
\bar{t}_{0}= & 0, \bar{s}_{0}=\bar{t}_{0}+\theta \eta, \\
\bar{t}_{1}= & \bar{s}_{0}+\left[\frac{\mid 1-\theta}{\left|\theta^{3}\right|}\left(1+\left|1-\theta^{2}\right|\right)+\frac{\left(\bar{s}_{0}-\bar{t}_{0}\right) K_{0}}{2 \theta^{2}}\right]\left(\bar{s}_{0}-\bar{t}_{0}\right), \\
\bar{s}_{1}= & \bar{t}_{1}+\frac{|\theta|}{1-K_{0} \bar{t}_{1}}\left[\frac{K}{2} \frac{\left|\theta^{2}-1\right|}{\theta^{2}}\left(\bar{s}_{0}-\bar{t}_{0}\right)^{2} \frac{K}{2}\left(\bar{t}_{1}-\bar{s}_{0}\right)^{2}+K_{0}\left(\bar{s}_{0}-\bar{t}_{0}\right)\left(\bar{t}_{1}-\bar{s}_{0}\right)\right], \\
\bar{s}_{n+1}= & \bar{t}_{n+1}+\frac{|\theta|}{1-K_{0} \bar{t}_{n+1}}\left[\frac{\left|\theta^{2}-1\right|}{2 \theta^{2}}\left(\bar{s}_{n}-\bar{t}_{n}\right)^{2} \frac{\left(\bar{t}_{n+1}-\bar{s}_{n}\right)^{2}}{2}+\left(\bar{s}_{n}-\bar{t}_{n}\right)\left(\bar{t}_{n+1}-\bar{s}_{n}\right)\right], \\
\bar{t}_{n+2}= & \bar{s}_{n+1}+\frac{K}{\theta^{2}\left(1-K_{0} \bar{t}_{n+1}\right)}\left\{| 1 - \theta | ( 1 + | 1 - \theta ^ { 2 } | ) \left[\frac{\left|\theta^{2}-1\right|}{2 \theta^{2}}\left(\bar{s}_{n}-\bar{t}_{n}\right)^{2} \frac{\left(\bar{t}_{n+1}-\bar{s}_{n}\right)^{2}}{2}\right.\right. \\
& \left.\left.+\left(\bar{s}_{n}-\bar{t}_{n}\right)\left(\bar{t}_{n+1}-\bar{s}_{n}\right)\right] \frac{1}{2}\left(\bar{s}_{n+1}-\bar{t}_{n+1}\right)^{2}\right\} \text { for each } n=0,1,2, \ldots
\end{aligned}
$$


Then, a simple induction argument shows that

$$
\begin{aligned}
\bar{s}_{n} & \leq s_{n}, \\
\bar{t}_{n} & \leq t_{n}, \\
\bar{s}_{n}-\bar{t}_{n} & \leq s_{n}-t_{n}, \\
\bar{t}_{n+1}-\bar{s}_{n} & \leq t_{n+1}-s_{n},
\end{aligned}
$$

and

$$
\bar{t}^{*}=\lim _{n \rightarrow \infty} \bar{t}_{n} \leq t^{*} .
$$

Clearly, $\left\{\bar{t}_{n}\right\},\left\{\bar{s}_{n}\right\}, \bar{t}^{*}$ can replace $\left\{t_{n}\right\},\left\{s_{n}\right\}, t^{*}$ in Theorem 3.2.

(b) The limit point $t^{*}$ can be replaced by $t^{* *}$ given in closed form by (3.9).

(c) Criteria $(\triangle)$ or $(2.9)$ and (2.11) are sufficient for the convergence of the third order method (1.2). However, these criteria are not also necessary. In practice, we shall test to see which of these criteria are satisfied (if any) and then use the best possible error bounds and uniqueness results (see also the numerical examples in the next section).

\section{Numerical examples}

Example 4.1. Let $x \in D, X=Y=\mathbb{R}, x_{0}=1$ and $D=\bar{U}(1,1)$. Define function $F$ on $D$ by

$$
F(x)=x^{3}-0.49 \text {. }
$$

Then, we get that

$$
\beta=\frac{1}{3}, \quad \eta=0.17, \quad M=12 .
$$

Now choosing $\theta=1.15$ we obtain that

$$
a=0.68, \quad \alpha=0.68, \quad \gamma=0.34
$$

and as a consequence $a_{1} b_{1}=134.091 \leq 1$ condition $(2.9)$ is violated. Hence, there is no guarantee under the conditions given in [7] that sequence $\left\{x_{n}\right\}$ converges to $x^{*}$. Calculating now $\delta_{f}$ and $\delta_{g}$, the smallest solutions of the polynomials $f(t)$ and $g(t)$ given in (3.1) and (3.2) respectively between 0 and 1 , we obtain that

$$
\delta=\min \left\{\delta_{f}, \delta_{g}\right\}=.4104586 \cdots .
$$

Moreover, we observe that the $\Delta$ conditions are satisfied since

$$
\begin{gathered}
\left|\frac{1-\theta}{\theta^{3}}\right|\left(1+\left|1-\theta^{2}\right|\right)+\frac{K \eta}{2 \theta}=.278261 \cdots \leq \delta, \\
0<\frac{K|\theta|}{1-K_{0}(1+\delta) s_{0}}\left[\frac{\left|\theta^{2}-1\right|}{2 \theta^{2}}+\frac{\delta^{2}}{2}+\delta\right]\left(s_{0}-t_{0}\right)=.360324 \cdots \leq \delta
\end{gathered}
$$

and

$0<\frac{K}{\theta^{2}\left(1-K_{0}(1+\delta) s_{0}\right)}\left\{|1-\theta|\left(1+\left|1-\theta^{2}\right|\right)\left[\frac{\left|\theta^{2}-1\right|}{2 \theta^{2}}+\frac{\delta^{2}}{2}+\delta\right]+\frac{\delta^{2}}{2}\right\}\left(s_{0}-t_{0}\right)$ 


$$
=.136162 \cdots \leq .168476 \cdots=\delta^{2} .
$$

Consequently, convergence to the solution is guaranteed by Theorem 3.2. Moreover, the computational order of convergence (COC) is shown in Table 1. Here $(\mathrm{COC})$ is defined by

$$
\rho \approx \ln \left(\frac{\left\|\bar{x}_{n+1}-\bar{x}^{\star}\right\|_{\infty}}{\left\|\bar{x}_{n}-\bar{x}^{\star}\right\|_{\infty}}\right) / \ln \left(\frac{\left\|\bar{x}_{n}-\bar{x}^{\star}\right\|_{\infty}}{\left\|\bar{x}_{n-1}-\bar{x}^{\star}\right\|_{\infty}}\right), \quad n \in \mathbb{N},
$$

Table 1 shows the (COC).

TABle 1. COC for Example 1 using $\theta=1.15$.

\begin{tabular}{lc}
\hline$n$ & $C O C$ \\
\hline 1 & 2.73851 \\
2 & 2.99157 \\
3 & 2.99999 \\
4 & 3.00000 \\
5 & 3.00000 \\
\hline$\rho=3.00000$ &
\end{tabular}

Example 4.2. Let $X=Y=\mathcal{C}[0,1]$, the space of continuous functions defined in $[0,1]$ equipped with the max-norm. Let $\Omega=\{x \in \mathcal{C}[0,1] ;\|x\| \leq R\}$, such that $R>1$ and $F$ defined on $\Omega$ and given by

$$
F(x)(s)=x(s)-f(s)-\lambda \int_{0}^{1} G(s, t) x(t)^{3} d t, \quad x \in C[0,1], s \in[0,1],
$$

where $f \in \mathcal{C}[0,1]$ is a given function, $\lambda$ is a real constant and the kernel $G$ is the Green function

$$
G(s, t)= \begin{cases}(1-s) t, & t \leq s, \\ s(1-t), & s \leq t .\end{cases}
$$

In this case, for each $x \in \Omega, F^{\prime}(x)$ is a linear operator defined on $\Omega$ by the following expression:

$$
\left[F^{\prime}(x)(v)\right](s)=v(s)-3 \lambda \int_{0}^{1} G(s, t) x(t)^{2} v(t) d t, \quad v \in C[0,1], s \in[0,1] .
$$

If we choose $x_{0}(s)=f(s)=1$, it follows $\left\|I-F^{\prime}\left(x_{0}\right)\right\| \leq 3|\lambda| / 8$. Thus, if $|\lambda|<8 / 3, F^{\prime}\left(x_{0}\right)^{-1}$ is defined and

$$
\left\|F^{\prime}\left(x_{0}\right)^{-1}\right\| \leq \frac{8}{8-3|\lambda|}
$$

Moreover,

$$
\begin{gathered}
\left\|F\left(x_{0}\right)\right\| \leq \frac{|\lambda|}{8} \\
\left\|F^{\prime}\left(x_{0}\right)^{-1} F\left(x_{0}\right)\right\| \leq \frac{|\lambda|}{8-3|\lambda|} .
\end{gathered}
$$


On the other hand, for $x, y \in \Omega$ we have

$$
\left[\left(F^{\prime}(x)-F^{\prime}(y)\right) v\right](s)=3 \lambda \int_{0}^{1} G(s, t)\left(x(t)^{2}-y^{2}(t)\right) v(t) d t
$$

and for $x \in \Omega$ we get in turn that

$$
\left\|F^{\prime \prime}(x)\right\| \leq \frac{6|\lambda|}{8} .
$$

Consequently,

$$
\begin{gathered}
\left\|F^{\prime}(x)-F^{\prime}(y)\right\| \leq\|x-y\| \frac{3|\lambda|(\|x\|+\|y\|)}{8} \leq\|x-y\| \frac{6 R|\lambda|}{8}, \\
\left\|F^{\prime}(x)-F^{\prime}(1)\right\| \leq\|x-1\| \frac{1+3|\lambda|(\|x\|+1)}{8} \leq\|x-1\| \frac{1+3(1+R)|\lambda|}{8} .
\end{gathered}
$$

Choosing $\lambda=1.5, R=4.4$ and $\theta=1.1$ we have

$$
\begin{gathered}
\beta=0.677966 \cdots, \\
\eta=0.127119 \cdots, \\
M=4.95, \\
a=0.426602 \cdots, \\
\alpha=1.16529 \cdots,
\end{gathered}
$$

and

$$
\gamma=0.213301 \cdots
$$

So, as $a_{1} b_{1}=1.25402 \leq 1$, condition (2.9) is violated. Hence, there is no guarantee under the conditions given in [7] that sequence $\left\{x_{n}\right\}$ converges to $x^{*}$. Calculating now $\delta_{f}$ and $\delta_{g}$, the smallest solutions of the polynomials $f(t)$ and $g(t)$ given in (3.1) and (3.2) respectively between 0 and 1, we obtain that

$$
\delta=\min \left\{\delta_{f}, \delta_{g}\right\}=0.370693 \cdots .
$$

Moreover, we observe that the $\Delta$ conditions are satisfied since

$$
\begin{gathered}
\left|\frac{1-\theta}{\theta^{3}}\right|\left(1+\left|1-\theta^{2}\right|\right)+\frac{K \eta}{2 \theta}=0.284819 \cdots \leq \delta, \\
0<\frac{K|\theta|}{1-K_{0}(1+\delta) s_{0}}\left[\frac{\left|\theta^{2}-1\right|}{2 \theta^{2}}+\frac{\delta^{2}}{2}+\delta\right]\left(s_{0}-t_{0}\right)=0.334767 \cdots \leq \delta
\end{gathered}
$$

and

$$
\begin{gathered}
0<\frac{K}{\theta^{2}\left(1-K_{0}(1+\delta) s_{0}\right)}\left\{|1-\theta|\left(1+\left|1-\theta^{2}\right|\right)\left[\frac{\left|\theta^{2}-1\right|}{2 \theta^{2}}+\frac{\delta^{2}}{2}+\delta\right]+\frac{\delta^{2}}{2}\right\}\left(s_{0}-t_{0}\right) \\
=0.0871515 \cdots \leq 0.137413 \cdots=\delta^{2} .
\end{gathered}
$$

Consequently, convergence to the solution is guaranteed by Theorem 3.2.

Acknowledgement. The research is partly supported by the grant MTM201128636-C02-01. 


\section{References}

[1] S. Amat, Á. A. Magreñán, and N. Romero, On a two-step relaxed Newton-type method, App. Math. Comput. 219 (2013), no. 24, 11341-11347.

[2] I. K. Argyros, Computational theory of iterative methods, Series: Studies in Computational Mathematics, 15, Editors: C. K. Chui and L. Wuytack, Elsevier Publ. Co. New York, U.S.A, 2007.

[3] I. K. Argyros and S. Hilout, Weaker conditions for the convergence of Newton's method, J. Complexity 28 (2012), no. 3, 364-387.

[4] — Computational Methods in Nonlinear Analysis, World Scientific Publ. Comp., New Jersey, 2013.

[5] V. Candela and A. Marquina, Recurrence relations for rational cubic methods I: The Halley method, Computing 44 (1990), no. 2, 169-184.

[6] - Recurrence relations for rational cubic methods II: The Chebyshev method, Computing 45 (1990), no. 4, 355-367.

[7] C. Chun, P. Stanica, and B. Neta, Third order family of methods in Banach spaces, Comput. Math. Appl. 61 (2011), no. 6, 1665-1675.

[8] J. A. Ezquerro and M. A. Hernández, Recurrence relations for Chebyshev-type methods, Appl. Math. Optim. 41 (2000), no. 2, 227-236.

[9] J. M. Gutiérrez and M. A. Hernández, Recurrence relations for the super-Halley method, Computers Math. Appl. 36 (1998), no. 7, 1-8.

[10] - Third-order iterative methods for operators with bounded second derivative, J. Comput. Appl. Math. 82 (1997), no. 1-2, 171-183.

[11] J. M. Gutiérrez, Á. A. Magreñán, and N. Romero, On the semilocal convergence of Newton-Kantorovich method under center-Lipschitz conditions, Appl. Math. Comput. 221 (2013), 79-88.

[12] L. V. Kantorovich and G. P. Akilov, Functional Analysis, Pergamon Press, Oxford, 1982.

[13] J. S. Kou and T. Li, Modified Chebyshev's method free from second derivative for nonlinear equations, Appl. Math. Comput. 187 (2007), no. 2, 1027-1032.

[14] J. S. Kou, T. Li, and X. H. Wang, A modification of Newton method with third-order convergence, Appl. Math. comput. 181 (2006), no. 2, 1106-1111.

[15] Á. A. Magreñán, Estudio de la dinámica del método de Newton amortiguado, $\mathrm{PhD}$ Thesis, Servicio de Publicaciones, Universidad de La Rioja, 2013.

[16] Á. A. Magreñán and I. K. Argyros, Two-step Newton methods, J. Complexity 30 (2014), no. 4, 533-553.

[17] J. M. Ortega and W. C. Rheinboldt, Iterative Solution of Nonlinear Equations in Several Variables, Academic press, New York, 1970.

[18] P. K. Parida, Study of some third order methods for nonlinear equations in Banach spaces, Ph.D. Dessertation, Indian Institute of Technology, Department of Mathematics, Kharagpur, India, 2007.

[19] P. K. Parida and D. K. Gupta, Recurrence relations for semilocal convergence of a Newton-like method in Banach spaces, J. Math. Anal. Appl. 345 (2008), 350-361.

[20] F. A. Potra and V. Ptră, Nondiscrete induction and iterative processes, in Research Notes in Mathematics, Vol. 103, Pitman, Boston, 1984.

[21] L. B. Rall, Computational solution of nonlinear operator equations, Robert E. Krieger, New York, 1979

[22] Q. Wu and Y. Zhao, Third order convergence theorem by using majorizing function for a modified Newton method in Banach space, Appl. Math. Comput. 175 (2006), no. 2, $1515-1524$. 
IOANNiS KONSTANTINOS ARGYRo

Department of Mathematical Sciences

CAMERon University

LAWTON, OK 73505, USA

E-mail address: iargyros@cameron.edu

Santhosh George

Department of Mathematical and Computational Sciences

National Institute of Technology

KARNATAKA, INDIA

E-mail address: sgeorge@nitk.ac.in

Ángel Alberto Magreñán

Departamento de TFG/TFM

UNIVERSIDAD INTERNACIONAL DE LA RIOJA (UNIR)

LOGROÑO, SPAIN

E-mail address: angel.magrenan@unir.net 